International Journal of Pure and Applied Mathematics

Volume 85 No. 5 2013, 955-963

ISSN: 1311-8080 (printed version); ISSN: 1314-3395 (on-line version)

url: http://www.ijpam.eu

doi: http://dx.doi.org/10.12732/ijpam.v85i5.13

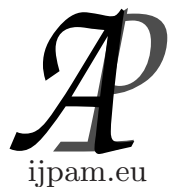

\title{
GENERALIZED VOLTERRA SPACES
}

\author{
Milan Matejdes \\ Department of Mathematics and Descriptive Geometry \\ Technical University in Zvolen \\ T.G. Masaryka 24, SK-960 53 Zvolen, SLOVAKIA \\ and \\ Department of Mathematics \\ Faculty of Applied Informatics \\ Tomas Bata University in Zlín \\ Nad Stráněmi 4511, CZ-760 05 Zlín, CZECH REPUBLIC
}

\begin{abstract}
In this paper we deals with a general concept of classification of subsets of a topological space $X$ with respect to a given nonempty system $\mathcal{E} \subset 2^{X} \backslash\{\emptyset\}$. Using system $\mathcal{E}$, the notions of $\mathcal{E}$-Volterra and weakly $\mathcal{E}$-Volterra spaces are introduced which covers classical Volterra and weakly Volterra spaces as well as irresolvable spaces.
\end{abstract}

AMS Subject Classification: 54A05, 54E52

Key Words: Baire space, Volterra space, weakly Volterra space, resolvable space

\section{Motivation and Basic Definitions}

In the sequel, $X$ is a nonempty topological space. By $\bar{A}, A^{\circ}$ we denote the closure, the interior of $A$, respectively and $\mathcal{U}(z)$ is a base of the open neighborhoods of a point $z$.

By [1], a topological space $X$ is weakly Volterra (Volterra), if for any $G_{\delta}$-sets $A_{1}$ and $A_{2}$ which are dense in $X, A_{1} \cap A_{2}$ is nonempty (dense in $X$ ).

Received: May 20, 2013

(c) 2013 Academic Publications, Ltd. url: www.acadpubl.eu 
It is clear, that any Baire space is Volterra and any space of second category is weakly Volterra. Recall that a space $X$ is Baire (of second category) if the intersection of any countably many dense open subsets is dense in $X$ (nonempty). In general, these four classes of spaces are all distinct and relevant examples can be found in [3], [4], [5], [6].

There are a few possibilities how to generalize the definitions mentioned above. Firstly, we can change the $G_{\delta}$-sets by the sets from a priori given system which are understood as "big". As for the systems, we can take into account many types of sets. For example: the nonempty open sets, the sets which are not nowhere dense, the sets of second category, the sets of second category with the Baire property, the perfect sets, the Borel sets, uncountable sets, the sets which are not from a given ideal, respectively.

The "bigness" of a set will be derived from the well known Kuratowski operator. Any nonempty system $\mathcal{E} \subset 2^{X} \backslash\{\emptyset\}$ will be called a cluster system in $X$. If any nonempty open subset of a nonempty open set $G$ contains a set from $\mathcal{E}$, then $\mathcal{E}$ is called a $\pi$-network in $G$. For a cluster system $\mathcal{E}$ and a subset $A$ of a topological space $X$, we can define the set $\mathcal{E}(A)$ of all points $x \in X$ such that for any neighborhood $U$ of $x$, the intersection $U \cap A$ contains a set form $\mathcal{E}$. Roughly speaking, $A$ is $\mathcal{E}$-big at a point $x$. Apart from, we will be interested in the complement of $A$, namely whether $X \backslash A$ is $\mathcal{E}$-big at a point $x$ or not. Our observation is based on the idea: A system $\left\{A_{t}\right\}_{t \in T}$ of pairwise disjoint sets is "small" near a point $x$ if $x \in \cap_{t \in T} \mathcal{E}\left(A_{t}\right)$ (there are "many" pairwise disjoint sets which are $\mathcal{E}$-big at $x)$. Similarly, if $A \subset \cap_{t \in T} \mathcal{E}\left(A_{t}\right)\left(\mathcal{E}(A) \subset \cap_{t \in T} \mathcal{E}\left(A_{t}\right)\right)$, then the system $\left\{A_{t}\right\}_{t \in T}$ is "small" near $A$ ("small" near points in which $A$ is $\mathcal{E}$-big). Further, we can ask and investigate some further qualitative properties of the intersection $\cap_{t \in T} A_{t}$. For example, it can be nonempty, dense in $X$, $\mathcal{E}\left(\cap_{t \in T} A_{t}\right) \neq \emptyset$ (it is $\mathcal{E}$-big at some point) or $\mathcal{E}\left(\cap_{t \in T} A_{t}\right)$ can have an appropriate topological property.

In this paper we focus on the research of the basic properties of weak $\mathcal{E}$ Volterra and $\mathcal{E}$-Volterra spaces which correspond with the known results of the weak Volterra, Volterra and irresolvable spaces. Other alternative systems mentioned above as well as other properties of $\cap_{t \in T} A_{t}$ are left for further research. We introduce the next definition.

Definition 1. A set $A$ is called weakly $\mathcal{E}$-Volterra, if for any two sets $A_{1}$ and $A_{2}$, such that $\mathcal{E}(A) \subset \mathcal{E}\left(A_{i}\right), i=1,2, A_{1} \cap A_{2}$ is nonempty. Moreover, if $A \neq \emptyset$ and $\overline{A_{1} \cap A_{2}} \supset A$, i.e., $A_{1} \cap A_{2}$ is dense in $A$, then $A$ is called $\mathcal{E}$-Volterra.

If $\mathcal{E}(A)=\emptyset$, then $A$ is not weakly $\mathcal{E}$-Volterra (see Remark 1 item (4)).

In the next example, we will give a few special cluster systems. Two of 
them (item (2) and (3)) describe irresolvable and weakly Volterra spaces. Their deeper investigation will be delivered in the next papers.

\section{Example 1.}

(1) Consider a cluster system $\mathcal{E}=\{G: G$ is nonempty open $\}$. Then $\mathcal{E}(A)=$ $\overline{A^{\circ}}$ and $\mathcal{E}(A)=\emptyset$ if and only if $A^{\circ}=\emptyset$. Let $\emptyset \neq \mathcal{E}(A) \subset \mathcal{E}\left(A_{i}\right), i=1,2$. Then $\emptyset \neq A^{\circ} \subset \overline{A^{\circ}} \subset \overline{A_{1}^{\circ}} \cap \overline{A_{2}^{\circ}}$, so $A_{1}^{\circ}$ and $A_{2}^{\circ}$ are open and dense in $A^{\circ}$. That means, $A_{1}^{\circ} \cap A_{2}^{\circ}$ is dense in $A^{\circ}$, so $A_{1} \cap A_{2}$ is dense in $A^{\circ}$. Hence $A_{1} \cap A_{2} \neq \emptyset$. Moreover, if $A$ is open, then $A_{1} \cap A_{2}$ is dense in $A$. We have proven $A$ is weakly $\mathcal{E}$-Volterra if and only if $A^{\circ} \neq \emptyset(A$ is not a boundary set) and any nonempty open set is $\mathcal{E}$-Volterra.

(2) By [2], a space $X$ is called resolvable if there are two disjoint subsets of $X$ which are dense in $X$ and $X$ is irresolvable if it is not resolvable. A subset $A$ of $X$ is resolvable (irresolvable) if $A$ as a subspace is resolvable (irresolvable). Finally, a space $X$ is hereditarily irresolvable if any nonempty subset of $X$ is irresolvable.

Put $\mathcal{E}_{0}=2^{X} \backslash\{\emptyset\}$. Then $\mathcal{E}_{0}(A)=\bar{A}$. Let $\mathcal{E}_{0}(X) \subset \mathcal{E}_{0}\left(A_{i}\right), i=1,2$. Then $X=\bar{X}=\mathcal{E}_{0}(X) \subset \mathcal{E}_{0}\left(A_{i}\right)=\overline{A_{i}}$, so $A_{1}$ and $A_{2}$ are dense in $X$. That means, $X$ is irresolvable (resolvable) if and only if $A_{1} \cap A_{2} \neq \emptyset$ $\left(A_{1} \cap A_{2}=\emptyset\right)$ for any (for some) $A_{1}$ and $A_{2}$ which are dense in $X$ or equivalently, $X$ is weakly $\mathcal{E}_{0}$-Volterra (not weakly $\mathcal{E}_{0}$-Volterra). Similarly, if $\mathcal{E}_{0}^{A}=2^{A} \backslash\{\emptyset\}, \emptyset \neq A \subset X$, then $A$ is irresolvable (resolvable) if and only if $A$ is weakly $\mathcal{E}_{0}^{A}$-Volterra (not weakly $\mathcal{E}_{0}^{A}$-Volterra). Finally, a space $X$ is hereditarily irresolvable if and only if any nonempty subset $A$ of $X$ is weakly $\mathcal{E}_{0}^{A}$-Volterra. Note, if $A^{\circ}=\emptyset$, then $A$ is not weakly $\mathcal{E}_{0}$-Volterra $(A \subset \bar{A}, A \subset \overline{X \backslash A}$ and $A \cap(X \backslash A)=\emptyset)$. For example, a singleton in the real line with usual topology is irresolvable but it is not weakly $\mathcal{E}_{0}$-Volterra. Moreover, if $X$ is resolvable, then any subset of $X$ is not weakly $\mathcal{E}_{0}$-Volterra (if $X_{1}, X_{2}$ are disjoint and dense in $X$, then for any $A$ we have $\bar{A}=\mathcal{E}_{0}(A) \subset \mathcal{E}_{0}\left(X_{i}\right)=\overline{X_{i}}=X, i=1,2$ and $\left.X_{1} \cap X_{2}=\emptyset\right)$.

(3) Let $E_{\delta}$ be a cluster system containing all $G_{\delta}$-sets and not nowhere dense. Then $X$ is weakly Volterra (Volterra) if and only if $X$ is weakly $\mathcal{E}_{\delta}$-Volterra $\left(\mathcal{E}_{\delta^{-}}\right.$-Volterra).

(4) Consider $X=\left\{0, \frac{1}{2}, \frac{1}{3}, \ldots, \frac{1}{n}, \ldots\right\}$ with the usual topology, $\mathcal{E}_{1}=\{A: A$ is infinite $\}$ and $\mathcal{E}_{2}=\{A: A$ is cofinite $\}$. Then $\mathcal{E}_{1}(X)=\mathcal{E}_{2}(X)=\{0\}$ and any subset of $X$ is not weakly $\mathcal{E}_{1}$-Volterra. 
On the other hand, $A \subset X$ is weakly $\mathcal{E}_{2}$-Volterra if and only if $A$ is cofinite. The sets $A_{1}=\left\{0, \frac{1}{2}, \frac{1}{4}, \frac{1}{6}, \ldots\right\}$ and $A_{2}=\left\{\frac{1}{3}, \frac{1}{5}, \frac{1}{7}, \ldots\right\}$ are not weakly $\mathcal{E}_{2}$-Volterra but $A_{1} \cup A_{2}=X$ is weakly $\mathcal{E}_{2}$-Volterra. There is no set which is $\mathcal{E}_{2}$-Volterra.

(5) A set $A$ is called preopen if there is an open set $G$ such that $A \subset G$ and $A$ is dense in $G(G \subset \bar{A})$, see [2]. Let $\mathcal{E}_{p}$ be a cluster system containing all nonempty preopen sets. Then $A$ is nowhere dense if and only if $\mathcal{E}_{p}(A)=\emptyset$ and $A \backslash \mathcal{E}_{p}(A)$ is nowhere dense. A space $X$ is weakly $\mathcal{E}_{p^{-}}$Volterra $\left(\mathcal{E}_{p^{-}}\right.$ Volterra) if and only if for any two dense sets in $X$ their intersection is nonempty, i.e., $X$ is irresolvable (dense in $X$ ).

\section{The Main Results}

We start with the basic properties of the operator $\mathcal{E}(A)$ and weakly $\mathcal{E}$-Volterra and $\mathcal{E}$-Volterra spaces. We omit or only outline the trivial proofs in the following remark.

\section{Remark 1.}

(1) $\mathcal{E}(A)$ is closed, $\mathcal{E}(A) \subset \bar{A}$ and if $A_{1} \subset A_{2}$, then $\mathcal{E}\left(A_{1}\right) \subset \mathcal{E}\left(A_{2}\right)$,

(2) Let $G$ be nonempty open. Then $\mathcal{E}$ is a $\pi$-network in $G$ if and only if $\mathcal{E}(G)=\mathcal{E}(\bar{G})=\bar{G}$

(3) If $A$ is weakly $\mathcal{E}$-Volterra ( $\mathcal{E}$-Volterra), then $A_{1} \cap A$ is nonempty $\left(A_{1} \cap A\right.$ is dense in $A$ ), for any $A_{1}$ such that $\mathcal{E}(A) \subset \mathcal{E}\left(A_{1}\right)$,

(4) If $\mathcal{E}(A)=\emptyset$ or $A=\emptyset$, then $A$ is not weakly $\mathcal{E}$-Volterra (for $A_{1}=A_{2}=\emptyset$, we have $\mathcal{E}(A)=\emptyset \subset \mathcal{E}\left(A_{1}\right)=\mathcal{E}\left(A_{2}\right)=\emptyset$ and $\left.A_{1} \cap A_{2}=\emptyset\right)$. Consequently, if $A$ is weakly $\mathcal{E}$-Volterra, then $\mathcal{E}(A)$ and $A$ are nonempty,

(5) If $A$ is weakly $\mathcal{E}$-Volterra (not weakly $\mathcal{E}$-Volterra), then any $B \supset A(B \subset$ $A)$ is weakly $\mathcal{E}$-Volterra (not weakly $\mathcal{E}$-Volterra),

(6) If $A$ is $\mathcal{E}$-Volterra, then $\bar{A}$ is $\mathcal{E}$-Volterra. (Let $A_{1}, A_{2}, i=1,2$, be such that $\mathcal{E}(\bar{A}) \subset \mathcal{E}\left(A_{i}\right)$. Since $\mathcal{E}(A) \subset \mathcal{E}(\bar{A})$ and $A$ is $\mathcal{E}$-Volterra, $A_{1} \cap A_{2}$ is dense in $A$, so it is dense in $\bar{A}$,

(7) If $\mathcal{E}$ is a $\pi$-network in a nonempty open set $G$, then $G$ is weakly $\mathcal{E}$-Volterra if and only if $\bar{G}$ is weakly $\mathcal{E}$-Volterra. (" $\Rightarrow$ " follows from item $(5)$. " $\Leftarrow "$ 
Let $A_{i}$ be such that $\mathcal{E}\left(A_{i}\right) \supset \mathcal{E}(G), i=1,2$. Since $\mathcal{E}(G)=\mathcal{E}(\bar{G})$ (see item $(2))$ and $\bar{G}$ is weakly $\mathcal{E}$-Volterra, $A_{1} \cap A_{2} \neq \emptyset$.),

(8) If $\mathcal{E}$ is a $\pi$-network in a nonempty open set $G$, then $G$ is $\mathcal{E}$-Volterra if and only if $\bar{G}$ is $\mathcal{E}$-Volterra. (" $\Rightarrow$ " follows from item (6). " $\Leftarrow$ " Let $A_{i}$ be such that $\mathcal{E}\left(A_{i}\right) \supset \mathcal{E}(G), i=1,2$. Since $\mathcal{E}(G)=\mathcal{E}(\bar{G})$ (see item $(2)$ ) and $\bar{G}$ is $\mathcal{E}$-Volterra, $A_{1} \cap A_{2}$ is dense in $\bar{G}$, so it is dense in $G$ ),

(9) If $A$ is not weakly $\mathcal{E}$-Volterra, then $\mathcal{E}(A)$ is also not weakly $\mathcal{E}$-Volterra (if $A_{1} \cap A_{2}=\emptyset$ and $\mathcal{E}(A) \subset \mathcal{E}\left(A_{i}\right), i=1,2$, then by item $(1), \mathcal{E}(\mathcal{E}(A)) \subset$ $\left.\overline{\mathcal{E}(A)}=\mathcal{E}(A) \subset \mathcal{E}\left(A_{i}\right)\right)$.

Theorem 1. (for nonempty weakly Volterra open subspase see [1], Theorem 2.3) Let $\mathcal{E}$ be a $\pi$-network in a nonempty open set $G$. If $G$ is weakly $\mathcal{E}$-Volterra, then for any two sets $A_{1}$ and $A_{2}$ such that $\mathcal{E}(G) \subset \mathcal{E}\left(A_{i}\right), i=1,2$, $A_{1} \cap A_{2} \cap G$ is not nowhere dense. Consequently, if $\mathcal{E}$ is a $\pi$-network in $X$ and $X$ is weakly $\mathcal{E}$-Volterra, then $A_{1} \cap A_{2}$ is not nowhere dense.

Proof. First, we will show that $A_{1} \cap A_{2}$ is not nowhere dense. Let $\mathcal{E}(G)=$ $\bar{G} \subset \mathcal{E}\left(A_{i}\right)$ and $X_{i}:=A_{i} \backslash A_{1} \cap A_{2}, i=1,2$. Suppose $A_{1} \cap A_{2}$ is nowhere dense. We will show $\mathcal{E}\left(X_{i}\right) \supset \mathcal{E}(G), i=1,2$. Let $x \in \mathcal{E}(G)=\bar{G}$ and $U \in \mathcal{U}(x)$. Then $U \cap G$ is nonempty, so there is a nonempty open set $H \subset U \cap G$ and $H \cap A_{1} \cap A_{2}=\emptyset$. Since $H \subset \bar{G} \subset \mathcal{E}\left(A_{i}\right)$, there is a set $E \in \mathcal{E}, E \subset H$ and $E \subset A_{i} \backslash A_{1} \cap A_{2}$, so $x \in \mathcal{E}\left(X_{i}\right) . G$ is weakly $\mathcal{E}$-Volterra, then $\emptyset \neq X_{1} \cap X_{2}=$ $\left(A_{1} \backslash A_{1} \cap A_{2}\right) \cap\left(A_{2} \backslash A_{1} \cap A_{2}\right)=\emptyset$, contradiction. Since for $B_{i}:=G \cap A_{i}$ the inclusion $\mathcal{E}(G) \subset \mathcal{E}\left(B_{i}\right)$ holds $(i=1,2), B_{1} \cap B_{2}=G \cap A_{1} \cap A_{2}$ is not nowhere dense.

By [1] (Lemma 3.3, Lemma 3.5), $X$ is Volterra if and only if any nonempty open subspace is weakly Volterra and the union of any family nonempty open non weakly Volterra subspace is not weakly Volterra. Similar results hold for a weakly $\mathcal{E}$-Volterra and $\mathcal{E}$-Volterra space.

Theorem 2. (for characterization of Volterra space see [1], Lemma 3.3 or [4]) If $\mathcal{E}$ is a $\pi$-network in an open set $X_{0}$, then $X_{0}$ is $\mathcal{E}$-Volterra if and only if any nonempty open subset of $X_{0}$ is weakly $\mathcal{E}$-Volterra.

Proof. Let $X_{0}$ be $\mathcal{E}$-Volterra. We will show that any nonempty open set $G \subset X_{0}$ is weakly $\mathcal{E}$-Volterra. Let $\mathcal{E}(G) \subset \mathcal{E}\left(A_{i}\right), i=1,2$. Since $X_{0}$ is $\mathcal{E}$ Volterra, there are two sets $X_{1}, X_{2}$, such that $\mathcal{E}\left(X_{1}\right) \supset \mathcal{E}\left(X_{0}\right), \mathcal{E}\left(X_{2}\right) \supset \mathcal{E}\left(X_{0}\right)$ 
and $X_{1} \cap X_{2}$ is dense in $X_{0}$. Let

$$
B_{i}=\left(G \cap A_{i}\right) \cup\left(\left(X_{0} \backslash \bar{G}\right) \cap X_{i}\right), i=1,2 .
$$

We will show $\mathcal{E}\left(B_{i}\right) \supset \mathcal{E}\left(X_{0}\right), i=1,2$. Let $x \in \mathcal{E}\left(X_{0}\right)=\overline{X_{0}} \subset \mathcal{E}\left(X_{i}\right)$. If $x$ is not from $\bar{G}$, then for any $U \in \mathcal{U}(x), U \cap \bar{G}=\emptyset$, there is a nonempty open set $H \subset U \cap X_{0} \subset \overline{X_{0}} \subset \mathcal{E}\left(X_{i}\right)$. So there is a set $E \in \mathcal{E}, E \subset U \cap X_{0} \cap X_{i}$. Since $E \cap \bar{G}=\emptyset, E \subset\left(X_{0} \backslash \bar{G}\right) \cap X_{i} \subset B_{i}$, so $x \in \mathcal{E}\left(B_{i}\right)$. If $x \in \bar{G}=\mathcal{E}(G) \subset \mathcal{E}\left(A_{i}\right)$, then for any $U \in \mathcal{U}(x), H:=U \cap G$ is nonempty and from inclusion $H \subset \bar{G}=$ $\mathcal{E}(G) \subset \mathcal{E}\left(A_{i}\right)$, there is a set $E \in \mathcal{E}$ such that $E \subset H \cap A_{i}=U \cap\left(G \cap A_{i}\right) \subset B_{i}$, so $x \in \mathcal{E}\left(B_{i}\right) . X_{0}$ is $\mathcal{E}$-Volterra, then $B_{1} \cap B_{2}$ is dense in $X_{0}$, so $B_{1} \cap B_{2} \cap G \neq \emptyset$. Then $\emptyset \neq B_{1} \cap B_{2} \cap G=G \cap A_{1} \cap A_{2}$, so $A_{1} \cap A_{2} \neq \emptyset$.

Suppose any nonempty open subset of $X_{0}$ is weakly $\mathcal{E}$-Volterra. Let $\mathcal{E}\left(A_{i}\right) \supset$ $\mathcal{E}\left(X_{0}\right), i=1,2$ and $G \subset X_{0}$ be nonempty open. Put $B_{i}:=A_{i} \cap G, i=1,2$. We will show $\mathcal{E}\left(B_{i}\right) \supset \mathcal{E}(G), i=1,2$. Let $x \in \mathcal{E}(G)=\bar{G}$ and $U \in \mathcal{U}(x)$ be arbitrary. Then $U \cap G \subset X_{0}$ is nonempty. Since any nonempty open subset of $X_{0}$ contains a set from $\mathcal{E}, U \cap G \subset \mathcal{E}\left(X_{0}\right) \subset \mathcal{E}\left(A_{i}\right)$. That means $U \cap G \cap A_{i}$ $\left(\subset B_{i}\right)$ contains a set from $\mathcal{E}$, so $\mathcal{E}\left(B_{i}\right) \supset \mathcal{E}(G)$. Since $G$ is weakly $\mathcal{E}$-Volterra, $B_{1} \cap B_{2}=A_{1} \cap A_{2} \cap G$ is nonempty, so $A_{1} \cap A_{2}$ is dense in $X_{0}$.

Theorem 3. Let $\mathcal{E}$ be a $\pi$-network in a nonempty open subset $X_{0}$. If $\left\{G_{t}\right\}_{t \in T}$ is a family of open disjoint non weakly $\mathcal{E}$-Volterra subsets of $X_{0}$, then $\cup_{t \in T} G_{t}$ is not weakly $\mathcal{E}$-Volterra.

Proof. Since $G_{t}$ is not weakly $\mathcal{E}$-Volterra, there are two subsets $A_{t}, B_{t}$ such that $\mathcal{E}\left(A_{t}\right) \supset \mathcal{E}\left(G_{t}\right), \mathcal{E}\left(B_{t}\right) \supset \mathcal{E}\left(G_{t}\right)$ and $A_{t} \cap B_{t}=\emptyset$. We will show $\mathcal{E}\left(A_{t} \cap G_{t}\right) \supset$ $\mathcal{E}\left(G_{t}\right)$ and $\mathcal{E}\left(B_{t} \cap G_{t}\right) \supset \mathcal{E}\left(G_{t}\right)$. Let $x \in \mathcal{E}\left(G_{t}\right)=\overline{G_{t}}$ (see Remark 1 item (2)) and $U$ be an arbitrary open neighborhood of $x$. Since $\mathcal{E}\left(A_{t}\right) \supset \mathcal{E}\left(G_{t}\right)=\overline{G_{t}} \supset G_{t} \cap U$ and $U \cap G_{t} \neq \emptyset$, there is a set $E \in \mathcal{E}, E \subset A_{t} \cap U \cap G_{t}$, so $x \in \mathcal{E}\left(A_{t} \cap G_{t}\right)$. Similarly for $\mathcal{E}\left(B_{t} \cap G_{t}\right) \supset \mathcal{E}\left(G_{t}\right)$. Let $C_{1}:=\cup_{t \in T}\left(A_{t} \cap G_{t}\right), C_{2}:=\cup_{t \in T}\left(B_{t} \cap G_{t}\right)$. We will show $\mathcal{E}\left(C_{i}\right) \supset \mathcal{E}\left(\cup_{t \in T} G_{t}\right), i=1,2$. Let $x \in \mathcal{E}\left(\cup_{t \in T} G_{t}\right)$ and $U$ be an arbitrary neighborhood of $x$. Then there is $t_{0}$ such that $U \cap G_{t_{0}} \neq \emptyset$. Let $i=1$. Since $\mathcal{E}\left(A_{t_{0}} \cap G_{t_{0}}\right) \supset \mathcal{E}\left(G_{t_{0}}\right)=\overline{G_{t_{0}}} \supset U \cap G_{t_{0}}$, there is $E \in \mathcal{E}$, $E \subset U \cap A_{t_{0}} \cap G_{t_{0}} \subset \cup_{t \in T}\left(A_{t} \cap G_{t}\right)=C_{1}$. Hence $x \in \mathcal{E}\left(C_{1}\right)$. Similarly for $i=2$. We have two sets $C_{1}$ and $C_{2}$ for which $\mathcal{E}\left(C_{i}\right) \supset \mathcal{E}\left(\cup_{t \in T} G_{t}\right), i=1,2$ and $C_{1} \cap C_{2}=\emptyset$, hence $\cup_{t \in T} G_{t}$ is not weakly $\mathcal{E}$-Volterra.

Remark 2. Let $\left\{G_{t}\right\}_{t \in T}$ be a family of nonempty open subsets of $X$. Using Zorn' lemma, there is a family $\mathcal{G}$ of pairwise disjoint open sets such that for each set $G \in \mathcal{G}$ there is a set $G_{t}$ such that $G \subset G_{t}$ and $\cup_{t \in T}\left\{G_{t}\right\} \subset \overline{\cup \mathcal{G}}$. 
Theorem 4. (for weakly Volterra open subspaces see [1], Lemma 3.5) Let $\left\{G_{t}\right\}_{t \in T}$ be a family of open non weakly $\mathcal{E}$-Volterra subsets of $X_{0}$. If $\mathcal{E}$ is a $\pi$-network in $X_{0}$, then $\overline{\cup_{t \in T} G_{t}}$ is not weakly $\mathcal{E}$-Volterra.

Proof. Let $\mathcal{G}$ be from Remark 2. By Theorem 3, $\cup \mathcal{G}$ is not weakly $\mathcal{E}$ Volterra. By Remark 1 item $(7), \overline{\cup \mathcal{G}}$ is not weakly $\mathcal{E}$-Volterra. Since $\overline{\cup_{t \in T}\left\{G_{t}\right\}} \subset$ $\overline{\cup \mathcal{G}}, \overline{\cup_{t \in T}\left\{G_{t}\right\}}$ is not weakly $\mathcal{E}$-Volterra, by Remark 1 item (5).

In [1] (Lemma 3.6), the decomposition of $X$ was given. Namely there are two open disjoint subspaces $X_{N V}, X_{V}$, such that $X=\overline{X_{N V}} \cup \overline{X_{V}}$, any nonempty open subspace of $X_{N V}$ is not weakly Volterra and any nonempty open subspace of $X_{V}$ is Volterra. Moreover, $X$ is Volterra if and only if $X_{N V}=\emptyset$ and $X$ is weakly Volterra if and only if $X_{V} \neq \emptyset$. The similar theorem holds for $\mathcal{E}$-Volterra and weakly $\mathcal{E}$-Volterra space.

Theorem 5. For any topological space $X$ there is a family $\mathcal{G}$ of pairwise disjoint nonempty open subsets of $X \backslash \mathcal{E}(X)$ (if $\mathcal{E}(X)=X$, then $\mathcal{G}=\emptyset$ ) and there are two disjoint open subsets $X_{0}, X_{1}$ of $\mathcal{E}(X)$ (both of them can be empty, see Example 1 item (4)) such that

(1) for any $G \in \mathcal{G}, G$ does not contain a set from $\mathcal{E}$ (that means, $G$ is not weakly $\mathcal{E}$-Volterra) and $\cup \mathcal{G}$ is dense in $X \backslash \mathcal{E}(X)$,

(2) $\overline{X_{0}}$ is not weakly $\mathcal{E}$-Volterra, so any subset of $\overline{X_{0}}$ is not weakly $\mathcal{E}$-Volterra,

(3) if $X_{1} \neq \emptyset$, then $\overline{X_{1}}$ is $\mathcal{E}$-Volterra and any nonempty open subset of $\overline{X_{1}}$ is $\mathcal{E}$-Volterra,

(4) $\mathcal{E}(X) \backslash\left(X_{0} \cup X_{1}\right)$ is nowhere dense, $\overline{X_{0}} \cap X_{1}=X_{0} \cap \overline{X_{1}}=\emptyset$.

Moreover, if $\mathcal{E}(X)=X$, then

(5) $X$ is $\mathcal{E}$-Volterra if and only if $\overline{X_{1}}=X$ or $X_{0}=\emptyset$,

(6) $X$ is weakly $\mathcal{E}$-Volterra if and only if $X_{1} \neq \emptyset$,

(7) Denote $F:=\overline{X_{0}}$ and $G:=X \backslash F$. Then $X=F \cup G$ is a decomposition of $X$, where $F$ is closed non weakly $\mathcal{E}$-Volterra (so any subset of $F$ is not weakly $\mathcal{E}$-Volterra) and if $G \neq \emptyset$, then any nonempty open subset of $G$ is $\mathcal{E}$-Volterra. 
Proof. (1) If $x \in X \backslash \mathcal{E}(X)$, then there is an open neighborhood $G$ of $x$, $G \subset X \backslash \mathcal{E}(X)$, such that $G$ does not contain a set from $\mathcal{E}$. Let $\left\{G_{t}\right\}_{t \in T}$ be a family of all nonempty open subsets of $X \backslash \mathcal{E}(X)$ such that for any $t \in T, G_{t}$ does not contains a set from $\mathcal{E}$. It is clear, $\cup_{t \in T} G_{t}=X \backslash \mathcal{E}(X)$. Let $\mathcal{G}$ be from Remark 2. Then $\mathcal{G}$ is a family of pairwise disjoint open sets, any open set $G$ from $\mathcal{G}$ does not contain a set from $\mathcal{E}$ (so $\mathcal{E}(G)=\emptyset$ and by Remark 1 item (4), $G$ is not weakly $\mathcal{E}$-Volterra) and $\cup \mathcal{G}$ is dense in $\cup_{t \in T} G_{t}=X \backslash \mathcal{E}(X)$, so item (1) holds.

Let $\mathcal{S}_{0}=\{G: G$ be an open non weakly $\mathcal{E}$-Volterra subset of $\mathcal{E}(X)\}$ and $\mathcal{S}_{1}=\{G: G$ be an open $\mathcal{E}$-Volterra subset of $\mathcal{E}(X)\}$. Put $X_{0}=\cup \mathcal{S}_{0}$ and $X_{1}=\cup \mathcal{S}_{1}$. Note, any nonempty open subset of $X_{i}$ contains a set from $\mathcal{E}$, so $\mathcal{E}$ is a $\pi$-network in $X_{i}, i=1,2$. Moreover, $X_{0} \cap X_{1}=\emptyset$. If not, there are two nonempty open sets $G_{0} \in \mathcal{S}_{0}$ and $G_{1} \in \mathcal{S}_{1}$ such that $\emptyset \neq G_{0} \cap G_{1}$. Then $G_{0} \cap G_{1}$ is not weakly $\mathcal{E}$-Volterra (as a subset of non weakly $\mathcal{E}$-Volterra set $G_{0}$, by Remark 1 item (5)) as well as weakly $\mathcal{E}$-Volterra (as a nonempty open subset of $G_{1}$ which is $\mathcal{E}$-Volterra, by Theorem 2), contradiction. So $X_{0} \cap X_{1}=\emptyset$.

(2) From Theorem $4, \overline{X_{0}}$ is not weakly $\mathcal{E}$-Volterra.

(3) Let $X_{1} \neq \emptyset$ and $G$ be a nonempty open subset of $\overline{X_{1}}$. We will show $G$ is $\mathcal{E}$-Volterra. Let $H$ be a nonempty open subset of $G$. Since $\overline{X_{1}} \backslash X_{1}$ is not nowhere dense, $H \cap X_{1} \neq \emptyset$ and $H \cap X_{1}$ is weakly $\mathcal{E}$-Volterra (if not, $H \cap X_{1} \subset X_{0}$, that means $X_{1} \cap X_{2} \neq \emptyset$, contradiction). That means $H$ is weakly $\mathcal{E}$-Volterra (see Remark 1 item (5)). We have proven any nonempty open subset $H$ of $G$ is weakly $\mathcal{E}$-Volterra, so by Theorem $2, G$ is $\mathcal{E}$-Volterra.

Since any nonempty open subset of $\bar{X}_{1}$ is $\mathcal{E}$-Volterra, $X_{1}$ is also $\mathcal{E}$-Volterra. Consequently, $\overline{X_{1}}$ is $\mathcal{E}$-Volterra, by Remark 1 item (6).

(4) Suppose $\mathcal{E}(X) \backslash\left(X_{0} \cup X_{1}\right)$ is not nowhere dense. Then there is a nonempty open set $G \subset \mathcal{E}(X) \backslash\left(X_{0} \cup X_{1}\right)$. We will show $G$ is $\mathcal{E}$-Volterra. Let $H \subset$ $G$ be nonempty open set. Then $H$ is weakly $\mathcal{E}$-Volterra (if not, $H \subset X_{0}$, contradiction), so $G$ is $\mathcal{E}$-Volterra, by Theorem 2 . Hence $G \subset X_{1}$, contraditcion. Let $\overline{X_{0}} \cap X_{1} \neq \emptyset$ or $X_{0} \cap \overline{X_{1}} \neq \emptyset$. Then $X_{0} \cap X_{1} \neq \emptyset$, contradiction.

Suppose $\mathcal{E}(X)=X$.

(5) If $\overline{X_{1}}=X$, then $X$ is $\mathcal{E}$-Volterra, by item (3). If $X_{0}=\emptyset$, then (by item (4)) $\mathcal{E}(X) \backslash\left(X_{0} \cup X_{1}\right)=X \backslash X_{1}$ is nowhere dense, so $X_{1}$ is dense in $X$ and by item (3), $X$ is $\mathcal{E}$-Volterra.

On the other hand, if $X$ is $\mathcal{E}$-Volterra, by Theorem 2, any nonempty open subset is weakly $\mathcal{E}$-Volterra, so any nonempty open subset is $\mathcal{E}$-Volterra. That means, $X_{1}=X$ and $X_{0}=\emptyset$.

(6) If $X_{1}=\emptyset$, then (by item (4)) $\mathcal{E}(X) \backslash\left(X_{0} \cup X_{1}\right)=X \backslash X_{0}$ is nowhere 
dense, so $X_{0}$ is dense in $X$ and by item (2), $X$ is not weakly $\mathcal{E}$ Volterra.

On the other hand, if $X_{1} \neq \emptyset$, by item (3), $X_{1}$ is $\mathcal{E}$-Volterra, so $X_{1}$ is weakly $\mathcal{E}$-Volterra. By Remark 1 item $(5), X$ is weakly $\mathcal{E}$-Volterra.

(7) By item (2), $F$ is not weakly $\mathcal{E}$-Volterra. We will show, if $G \neq \emptyset$, then any nonempty open subset of $G$ is $\mathcal{E}$-Volterra. Let $H \subset G$ be nonempty open. Then $H \backslash X_{1}$ as a subset of $X \backslash\left(X_{0} \cup X_{1}\right)$ is nowhere dense, by item (4). So $H \cap X_{1}$ is dense in $H$. By item (3), $H \cap X_{1}$ is $\mathcal{E}$-Volterra. Since $\overline{H \cap X_{1}}=\bar{H}$, $H$ is $\mathcal{E}$-Volterra, by Remark 1 item (8).

\section{Acknowledgments}

Partially supported by APVV-0269-11.

\section{References}

[1] J. Cao, D. Gauld, Volterra spaces revisited, J. Aust. Math. Soc., 79 (2005), 61-76, doi: 10.1017/S1446788700009332.

[2] M. Ganster, Preopen sets and resolvable spaces, Kyungpook Math. J., 27 (1987), 135-143.

[3] D. Gauld, S. Greenwood, Z. Piotrowski, On Volterra spaces II, Ann. New York Acad. Sci., 806 (1996), 169-173, doi: 10.1111/j.17496632.1996.tb49167.x.

[4] D. Gauld, S. Greenwood, Z. Piotrowski, On Volterra spaces III: Topological operations, Topology Proc., 23 (1998), 167-182.

[5] D. Gauld, Z. Piotrowski, On Volterra spaces, Fast East J. Math. Sci., 1 (1993), 209-214, doi: 10.1111/j.1749-6632.1996.tb49167.x.

[6] G. Gruenhage, D. Lutzer, Baire and Volterra spaces, Proc. Amer. Math. Soc., 128 (2000), 3115-3124, doi: 10.1090/S0002-9939-00-05346-6. 
